\title{
Estruturas factivas E PADRões de EXTRAÇÃo*
}

(Factive Structures: syntactic patterns)

Marina R. A. AUGUSTO (Universidade Estadual de Campinas/Fapesp)

ABSTRACT: This paper investigates the relationship between structural and semantic properties of factive sentences and the pattern of extraction exhibited. It is argued that a classification as weak or strong island is unfeasible for what has been termed Factive Island. The kinds of structures allowed as factive complements are analyzed as well as their corresponding behavior concerning extraction. The common feature these structures show is their presuppositional character, which is derived from a selection requirement. I assume that factive predicates select a [+ specific] complement. The differences showed concerning extraction constitute a spontaneous effect from the structural way each construction may satisfy this requirement.

KEY-WORDS: generative theory, factivity, specificity, movement.

RESUmo: Este artigo investiga as propriedades estruturais e semânticas das construções factivas e a relação dessas propriedades com possibilidades de extração. Argumenta-se que a tradicional classificação dessa construção como ilha fraca, ou excepcionalmente como forte, no caso do grego moderno, é insustentável. Tomando-se o ponto comum às estruturas complemento de predicados factivos, a saber, o seu caráter pressuposicional, assume-se que esses predicados selecionam um complemento do tipo [+específico]. A diversificação em relação ao padrão de extração se configura como um reflexo da maneira estrutural como esse requerimento é satisfeito. Palavras-Chave: gerativismo, factividade, especificidade, movimento.

\section{Introdução}

Este artigo trata das propriedades estruturais e semânticas das construções factivas e a relação dessas propriedades com possibilidades de extração. Argumenta-se que a distinção ilhas fortes/ilhas fracas - baseada no fato das estruturas pertencentes ao primeiro grupo (ilha do sujeito sentencial/ilha do adjunto) inviabilizarem qualquer tipo de movimento de elementos- $Q u$,

* A pesquisa que deu origem a este artigo se beneficiou dos comentários e sugestões de vários colegas. Agradeço à FAPESP pelo financiamento parcial. Meus agradecimentos especiais a Jairo Nunes e Mary Kato. 
enquanto as que pertencem ao segundo (entre as quais a ilha factiva, e também a ilha- $Q U$ ) admitirem a extração de argumentos, mas não a de adjuntos (ver Huang 1982, Chosmky 1986, Rizzi 1990, Cinque 1990) - não pode ser sustentada para a Ilha Factiva. Para essa construção, atesta-se uma aparente mobilidade entre comportamento de ilha fraca ou de ilha forte em uma mesma língua a depender do tipo de complemento factivo presente:

(1) O que o Pedro lamentou [que a Maria tenha feito $t_{i}$ ]?

(2) *Como o Pedro lamentou [que o mecânico tenha consertado o carro $t_{i}$ ]

(3) *O que o Pedro lamentou o fato de [que a Maria tenha feito $t_{i}$ ]?

(4) *Como o Pedro lamentou o fato de [que o mecânico tenha consertado o carro $\left.\mathrm{t}_{\mathrm{i}}\right]$ ?

A fim de conciliar a questão da característica semântica definidora dos predicados factivos, a saber, a de introduzirem a pressuposição de que a sentença encaixada expressa uma proposição verdadeira, e o quadro de extração que se mostra bem mais complexo do que o geralmente assumido em relação ao grupo das ilhas fracas, defendo que a Ilha Factiva não se caracteriza por uma estruturação sintática única, mas que diferentes estruturas se mostram adequadas para garantir a interpretação de pressuposto, sendo a diversidade de resultados obtidos em relação às possibilidades de extração um reflexo das restrições impostas por cada estrutura particular.

Na próxima seção, apresento as principais características assumidas em relação à Ilha Factiva e discuto o quadro de extração exibido por essa construção. A proposta para a conciliação entre característica semântica e propriedades de extração é apresentada na seção 3 , em que se propõe a presença de um nódulo TopP de caráter sentencial para um tipo de complementação factiva. A seção 4 expõe a proposta para o mapeamento da estrutura sintática a fim de se obter a interpretação semântica desejada. A seguir, apresentam-se dados adicionais que corroboram a análise proposta. A questão do grego moderno (língua que exibe um padrão peculiar em relação à extração na construção factiva) é exposta na seção 6. A última seção retoma os principais pontos e conclui o artigo.

\section{Principais características das construções factivas}

O trabalho de Kiparsky e Kiparsky (1971), que já se tornou um clássico, foi um dos pioneiros a listar uma classe de verbos denominados factivos, cujas relações semântico-sintáticas foram correlacionadas. Um dos principais diferenciadores dos factivos diz respeito à sua característica semântica de introduzirem a pressuposição de que a oração encaixada expressa uma 
proposição verdadeira. Segundo Kiparsky e Kiparsky (1971:348): “The speaker presupposes that the embedded clause expresses a true proposition, and makes some assertion about that proposition." A característica sintática marcante, tradicionalmente associada aos predicados factivos, apontada por Kiparsky e Kiparsky (1971), é a possibilidade de esses serem seguidos pelo sintagma $o$ fato:

(5) A população lamenta o fato (de) que o governo tenha agido fisiologicamente.

No entanto, conforme já mencionado, há distinções de gramaticalidade em relação à extração de argumentos quando da presença ou ausência desse sintagma nominal (cf. (1) e (3) acima), um tipo de dado que ainda não havia sido explorado satisfatoriamente nas análises acerca das construções factivas. Na seção 3.2 adiante, me detenho no tipo de relação estrutural entre o elemento "o fato" e a sentença que o segue, defendendo que esta é um aposto àquele.

Um ponto importante ainda a salientar em relação à presença desse sintagma acompanhando os verbos factivos é a obrigatoriedade do seu caráter definido (Kiparsky e Kiparsky 1971), haja vista a inaceitabilidade da sentença a seguir:

(6) *A população lamentou um fato de que o governo agira fisiologicamente.

Na verdade, a imposição que se dá pelos predicados factivos aos seus complementos é de que estes sejam específicos, ${ }^{2}$ conforme pode ser atestado por dados do tipo abaixo:

(7) a. *Pedro lamentou uma briga.

b. Pedro lamentou uma briga que ele teve com seu chefe.

(8) a. *Pedro lamentou um fato.

b. Pedro lamentou um fato que ocorreu ontem.

${ }^{1}$ O teste tradicional para se verificar se a proposição da oração complemento é pressuposta como verdadeira consiste em negar o predicado matriz como ilustrado a seguir:

(i) A população lamenta que o governo tenha agido fisiologicamente. (pressuposto: o governo agiu fisiologicamente)

(ii) A população não lamenta que o governo tenha agido fisiologicamente. (pressuposto: o governo agiu fisiologicamente)

2 Ihsane \& Puskás (2001:40) oferecem as definições a seguir a fim de contrapor definitude e especificidade: Definiteness: selects one object in the class of possible objects

Specificity: relates to pre-established elements in the discourse

As definições tomam por base Heim (1982) "definite serves to narrow down the range of things that can felicitously be referred to" e Enç (1991) "specificity involves a weak link, that of being a subset of or standing in some recoverable relation to a familiar object". 
Há uma necessidade de se especificar o sintagma complemento de lamentar, ou seja, sintagmas nominais indefinidos só são aceitáveis se modificados por uma relativa, uma vez que, conforme salientam Fodor e Sag (1982:36): "any relative clause modifying an indefinite adds to its descriptive content and thus tends to favor a referential understanding".

Enfim, verificamos que os predicados factivos impõem um caráter de especificidade ao seu complemento. Kiparsky e Kiparsky (1971:366), no apêndice ao seu artigo, especulam sobre a relação entre os conceitos de especificidade e verdade:

There is a syntactic and semantic correspondence between truth and specific reference. The verbs which presuppose that their sentential object expresses a true proposition also presuppose that their non-sentential object refers to a specific thing. (...) Perhaps this indicates that at some sufficiently abstract level of semantics, truth and specific reference are reducible to the same concept.

Avanço aqui uma proposta em que essas noções são unificadas.

Em relação à questão da extração, chamo a atenção para o seguinte quadro: ${ }^{3}$

\section{Inglês}

(9) What do you regret [that Mary bought $t_{i}$ on her trip to China]?

(10)*What ${ }_{i}$ do you regret the fact [that Mary bought $t_{i}$ on her trip to China]?

(11) *? What ${ }_{\mathrm{i}}$ do you regret it [that Mary bought ${ }_{\mathrm{i}}$ on her trip to China]?

(12) ${ }^{*} \mathrm{How}_{\mathrm{i}} / \mathrm{Why}_{\mathrm{i}}$ do you regret [that Mary fixed the broken vase $\mathrm{t}_{\mathrm{i}}$ ]?

(13) *?Where/When $n_{\mathrm{i}}$ do you regret [that Mary bought the vase $\mathrm{t}_{\mathrm{i}}$ ?

(14) *What the hell $\mathrm{i}_{\mathrm{i}}$ do you regret [that Mary bought $\mathrm{t}_{\mathrm{i}}$ on her trip to China]?

\section{Português}

(15) $\mathrm{O}_{\text {que }}$ você lamenta [que a Maria tenha comprado $t_{\mathrm{i}}$ na viagem à China]?

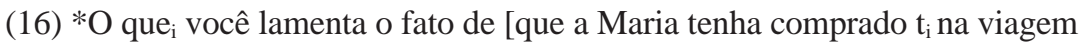
à China]?

(17) *Como $/$ Por que você lamenta [que a Maria tenha quebrado o vaso chinês $\left.\mathrm{t}_{\mathrm{i}}\right]$ ?

(18) *?Onde $/$ Quando ${ }_{i}$ você lamenta [que a Maria tenha quebrado o vaso chinês $\left.\mathrm{t}_{\mathrm{i}}\right]$ ?

\footnotetext{
3 A observação dos dados abaixo mostra que não estarei fazendo referência à extração de sujeito. Essa é uma questão mais geral em que se tem freqüentemente apontado o fato de que as línguas românicas permitem extração com maior facilidade. Em relação ao português, há uma distinção entre português brasileiro, que permite qualquer tipo de extração, e português europeu, para o qual se encontram algumas restrições (Zubizarreta 1982b). Essa distinção não será enfocada aqui.
} 


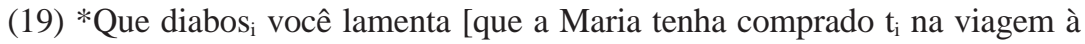
China]?

Os dados mostram que a questão da extração está longe de se conformar ao geralmente assumido em relação às ilhas fracas, isto é, extração de argumentos resultando gramatical, enquanto a extração de adjuntos é desautorizada.

É necessário, além de distinguir entre extração de argumentos e adjuntos, também explicar as distinções de gramaticalidade quando da presença explícita/lexical do NP o fato/the fact, ou do pronome it, no caso do inglês ${ }^{4}$. Em geral, podemos afirmar que a presença do sintagma nominal o fato induz a agramaticalidade mesmo para a extração de argumentos, ou seja, nesse contexto, a Ilha Factiva comporta-se como uma ilha forte, conforme atestam os exemplos (10) e (16) . Nos contextos em que a extração é possível, verificase possível melhora de aceitabilidade (variável entre falantes) para a extração dos denominados adjuntos referenciais (exemplos (13) e (18)): uma questão já debatida na literatura (Aoun, Hornstein, Lightfoot e Weinberg 1987, Rizzi, 1990, Cinque 1990) e intimamente ligada à noção de leitura D-linked de Pesetsky (1987). Os exemplos com o elemento interrogativo que diabos (14) e (19), fundamentalmente não $D$-linked, atestam a pertinência da relação.

A questão da impossibilidade de extração de argumentos em ilhas factivas remete a um outro ponto relevante nessa discussão, que diz respeito à variação interlinguística. Embora a Ilha Factiva seja geralmente tratada como uma ilha fraca, em que a extração de argumentos é possível, mas não a de adjuntos, seu comportamento é de ilha forte em algumas línguas, como, por exemplo, o grego moderno (Varlokosta 1994): ${ }^{6}$

\footnotetext{
4 Em relação ao português brasileiro, é preciso lembrar que já perdemos o clítico acusativo proposicional de terceira pessoa, totalmente em desuso no PB (Cyrino 1994), que seria o correspondente do it. Assim, sentenças como (i) abaixo, com a presença do pronome, são inaceitáveis. Vale, no entanto, salientar possibilidades como (ii) e (iii), exemplos fornecidos por Zubizarreta (1982a) e Raposo (1987) respectivamente, em que $o$ é um artigo definido e não um pronome átono acusativo de terceira pessoa, como em (i). O mesmo ocorre em espanhol (iv):

(i) *Eu o lamento imensamente que a Maria tenha comprado um vaso caro na sua viagem à China.

(ii) Pedro lamenta o terem-se lançado bombas.

(iii) Nós lamentamos o eles terem recebido pouco dinheiro.

(iv) Juan lamenta el que María haya comprado aquel cuadro en su viaje a China.

5 Conforme apontado anteriormente, assumo conforme exposição na seção 3.2, que a sentença que segue o elemento "o fato" é um adjunto (um aposto). Essa relação possivelmente possa se efetivar com alguns outros elementos distintos de "o fato", o que levaria ao estabelecimento de uma distinção entre completivas nominais e esse tipo de "complementação". Vale salientar que a completiva nominal tem se mostrado, com frequiência, problemática para as análises acerca das ilhas sintáticas (ver Augusto 1994).

${ }^{6}$ O complementizador que acompanha os verbos factivos em grego moderno pode ser grafado como ou pu. Embora opte pelo termo $p u$ na discussão que se segue, sou fiel à ortografia dos autores nas citações.
} 


\section{Grego Moderno}

(20) *Pjon lípase pou sinandise o Yanis?

Who regret-2.SG that met-3.SG John-NOM

Who do you regret that John met?

Outro ponto importante a ser considerado diz respeito às possibilidades de leitura permitidas para o elemento que sofre extração a partir da Ilha Factiva. Apenas a leitura referencial, isto é, a leitura de variável individual está disponível na extração a partir de ilhas em geral (Heycock 1995, Rizzi 2000). A interpretação não-referencial/intensional, disponível em extrações a partir de sentenças encaixadas de verbos não-factivos como dizer ou achar, por exemplo, não é possível. Para efeito de ilustração, vamos comparar as duas sentenças a seguir:

(21) Quantos filmes o Pedro acha que a Maria viu nas férias?
A: Três.
B: Esses três: Lavoura Arcaica, Copo de Cólera e Abril Despedaçado.
(22) Quantos filmes o Pedro lamenta que a Maria tenha visto nas férias

A: *Três.

B: Esses três: Lavoura Arcaica, Copo de Cólera e Abril Despedaçado.

A sentença (21) é ambígua. Há duas respostas possíveis: uma de quantidade (não-referencial) e outra referencial. Na primeira possibilidade, não se estará falando de quaisquer filmes específicos. Já para (22), há necessariamente um conjunto específico de filmes pressuposto. A interpretação de quantidade está excluída como resposta possível.

Outra distinção comumente reportada em relação a ilhas fracas diz respeito à possibilidade de leitura de lista de pares ou leitura individual (Szabolcsi e Zwarts 1992-3, Hornstein 1995). Essa distinção também se faz notar em sentenças com verbos factivos. Apenas a leitura individual é obtida, excluindo-se a leitura de lista de pares, possível com verbos não-factivos, como em (23):

(23) Quem os entrevistados disseram que o acusado matou?

A: Os entrevistados disseram que ele matou $X$ e $Y$.

B: $K$ disse que ele matou $X$. $L$ que ele matou $Y$ e $M$ que ele matou $Z$.

(24) Quem os entrevistados lamentaram que o acusado tenha matado?

A: Os entrevistados lamentaram que ele tenha matado $\mathrm{X}$ e $\mathrm{Y}$.

B: ${ }^{*} K$ lamentou que ele tivesse matado $X$. $L$ que ele tivesse matado $Y$ e $M$ que ele tivesse matado $Z$.

Em suma, as principais características da Ilha Factiva remetem, semanticamente, à propriedade de introduzir a pressuposição de que a sentença 
encaixada expressa uma proposição verdadeira. Em termos sintáticos, a Ilha Factiva faz parte do grupo das ilhas fracas, definidas como contextos estruturais a partir dos quais a extração de argumentos é possível, mas não a de adjuntos. No entanto, uma observação detalhada dos dados aponta para a inviabilidade de sustentação dessa distinção. Além de haver línguas em que o comportamento da Ilha Factiva não se conforma ao defendido para as ilhas fracas (no grego moderno, o comportamento é de ilha forte), verifica-se também a existência de estruturas que escapam à tradicional divisão caracterizadora do grupo das ilhas fracas. Por outro lado, é relevante apontar que, mesmo nos contextos em que a extração de argumentos resulta possível, há uma restrição de leitura que esses elementos sofrem.

\section{Uma proposta para a Ilha Factiva}

A discussão deixa claro que o corpo de dados apresentados até o momento não poderia ser coberto por nenhuma das análises anteriores de que se tem conhecimento (Kiparsky \& Kiparsky 1971, Melvold 1991, Hegarty 1992, Roussou 1994, Varlokosta 1994, Ormazabal 1995, Manzini 1997) ${ }^{7}$. A questão da extração se mostra mais complexa do que a distinção argumentos/adjuntos poderia abarcar. Isso me leva a defender estruturas distintas que possam implicar as distinções de extração observadas, um caminho que não foi amplamente explorado, dada a tendência em uniformizar as estruturas como reflexo de uma caracterização única para o fenômeno da factividade.

Alguns pontos interessantes de propostas anteriores são incorporados por esta análise. Um deles diz respeito ao caráter de tópico apresentado pela construção factiva e assumido por Manzini (1997) e outro diz respeito à noção de movimento do complemento, embutida na tese de Ormazabal (1995), que ganha uma nova concepção, mantendo-se a idéia de que a interpretação de contextos factivos e puramente proposicionais se deve às posições distintas que esses complementos ocupam. Essas noções são reinterpretadas na minha análise via a concepção de Estrutura de Asserção de Zubizarreta (1998).

Na seção anterior, chamei a atenção para o fato de que os complementos de predicados factivos não são exclusivamente elementos definidos, mas abarcam um grupo do qual os definidos podem ser considerados uma subclasse, isto é, os elementos específicos:

(25) *Pedro lamentou uma briga.

(26) Pedro lamentou uma briga que ele teve com seu chefe. 
A especificidade implica a identificação de indivíduos cuja existência é pressuposta (Diesing 1992). Analogamente, podemos dizer que essa mesma imposição se estende aos complementos sentenciais, ou seja, um complemento sentencial específico é aquele que identifica eventos cuja ocorrência é pressuposta. Essa aproximação entre complementos nominais e sentenciais abre caminho para a análise que defendo aqui em que se propõe que os predicados factivos impõem uma restrição de seleção aos seus complementos: apenas elementos específicos são admitidos. Essa especificidade do complemento é o que garante a leitura de pressuposto. Para tanto, estendo uma outra associação possível de ser traçada com os elementos passíveis de extração na Ilha Factiva. Conforme salientado, estes apresentam uma leitura referencial/específica. Seguindo Rizzi (2000), que associa os elementos passíveis de extração, em ilhas, à posição de tópico, proponho analogamente associar os complementos de predicados factivos a uma posição de tópico, a partir da implosão do nódulo CP adotada por Rizzi (1997). Assim, assumo a possibilidade de instanciação de um nódulo TopP sentencial. A adoção desse nódulo e a aplicação da Estrutura de Asserção de Zubizarreta (1998) me permitem obter o mapeamento desse complemento para uma posição na periferia esquerda da sentença, derivando, assim, a leitura de pressuposto ${ }^{8}$.

\subsection{Nódulo TopP sentencial}

Rizzi (1997) abre espaço para a incorporação de nódulos do tipo Tópico e Foco a partir da proposta de que o sistema CP seja desmembrado em vários constituintes. A proposta que estou fazendo é que, dados contextos particulares, a instanciação de um nódulo TopP com caráter sentencial poderá ser requerido. Isso se dá com os predicados factivos devido à imposição de seleção de complementos específicos. No caso de complementos sentenciais, a seleção de CP padrão não seria satisfatória, mas apenas a seleção de TopP sentencial, associado ao traço [+ específico] será capaz de satisfazer essa exigência. A idéia é estabelecer uma diferenciação entre sentenças complemento, estabelecendo-se a seguinte relação a nível sentencial: ${ }^{9}$

\footnotetext{
8 Ormazabal (1995) deriva a interpretação de pressuposto de um movimento do complemento factivo em LF, cogitando que o nódulo que o abrigaria talvez pudesse ser RefP (Beghelli e Stowell 1997). Remeto o leitor à seção 4.1, em que se discute a relevância de se tratar a pressuposição como um fenômeno em LF ou pós-LF.

9 Uma discussão acerca das noções de dicto e de re, assim como dos conceitos atributivo versus referencial em relação ao conceito de especificidade aqui tratado pode ser encontrada em Augusto (2003). Em termos gerais, "uma crença de dicto é uma crença cujo conteúdo é uma proposição completamente determinada, um dictum. Uma crença de re, em contraste, é uma crença cujo conteúdo é algo que de alguma maneira não chega a ser uma proposição completamente determinada; em particular, há na proposição uma menção a um objecto ou a uma coisa (res), mas não há qualquer especificação de um modo particular de identificação desse objecto pelo sujeito da crença. “(Enciclopédia de termos lógico-filosóficos)
} 
(27) Seleção de CP = leitura "de dicto"

Seleção de TopP = leitura "de re"

A projeção TopP sentencial conecta-se diretamente ao verbo factivo, sendo que o traço [+ específico] está em Top ${ }^{0}$. Ou seja, em termos simplificados, estamos assumindo que acima de CP propriamente dito, temse uma projeção TopP. O complementizador que é gerado em $\mathrm{C}^{0}$ ou Force ${ }^{0}$, a partir da implosão do CP por Rizzi (1997), sobe para TopP para checar o traço [+específico]:

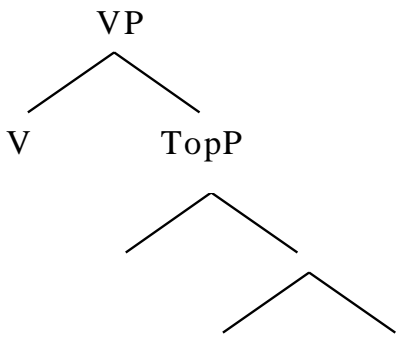

[+ específico] Force $\mathrm{P}$

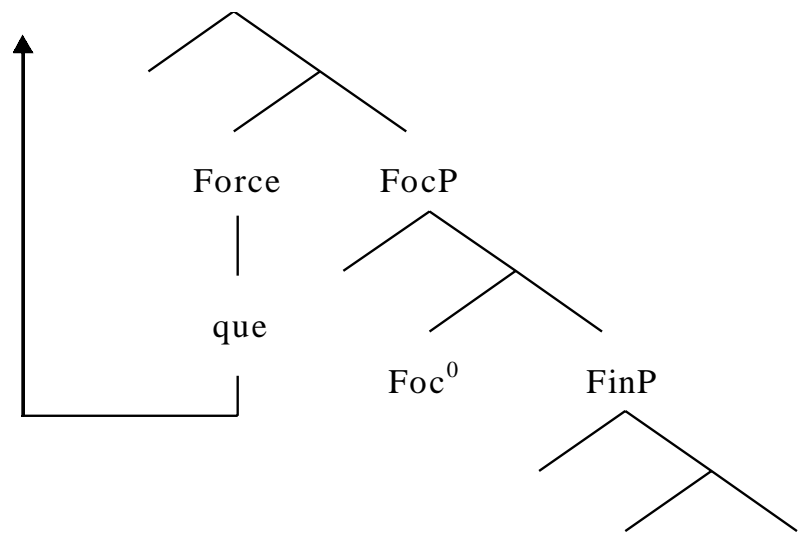

Fin $^{0}$

IP

Obtém-se, assim, a leitura de tópico sentencial, dado esse movimento do complementizador para Top ${ }^{0}$, estendendo-se o caráter de tópico para a sentença toda, o que acarreta a leitura de pressuposição.

Em relação à extração, o fato de TopP estar acima de CP implica algumas restrições. [Spec, TopP] se constitui como escape para o movimento de sintagmas- $Q u$, mas apenas elementos compatíveis com as caraterísticas da posição de tópico podem usar esse pouso intermediário, ou seja, somente 
argumentos, elementos $D$-linked. Adjuntos, por outro lado, não suportam uma interpretação compatível com aquela atribuída a [Spec, Top], logo, este especificador não pode recebê-los, o que inviabiliza movimentos subseqüientes que permitam que o elemento-Qu alcance a posição final, [Spec, CP] da matriz. A estrutura relevante é dada a seguir:

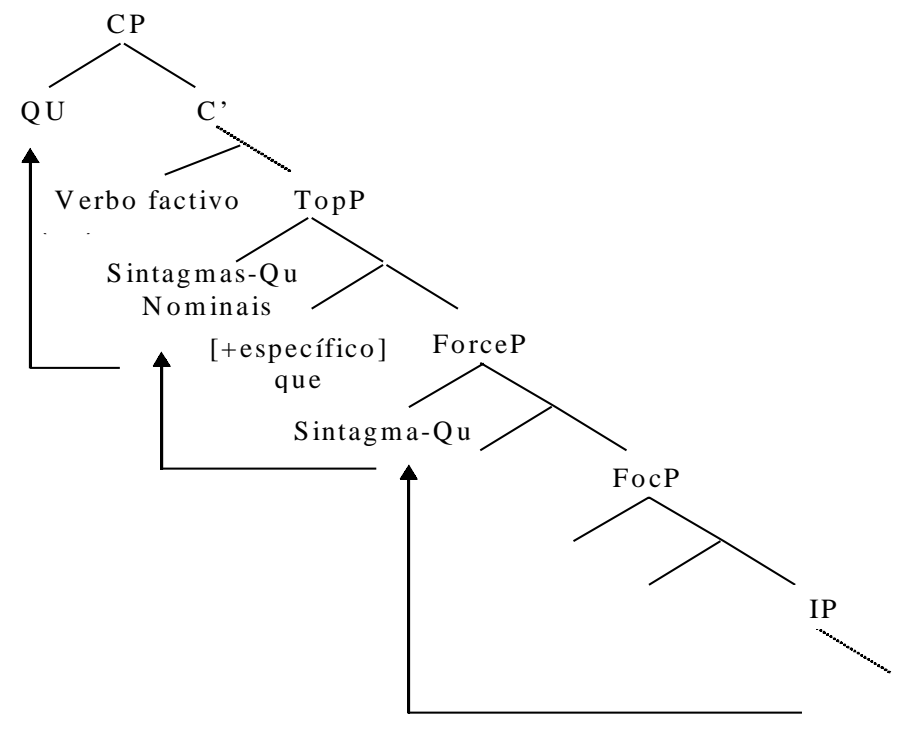

Em suma, a característica de especificidade/pressuposição do complemento de verbos factivos é alcançada pela presença do nódulo TopP e a relação que se estabelece entre a sentença complemento e esse nódulo, sendo as possibilidades de extração um reflexo dessa configuração estrutural proposta, ou seja, somente elementos compatíveis com as características associadas à posição de tópico poderão usar [Spec, TopP] como pouso intermediário para a extração. Isso exclui adjuntos e elementos intrinsecamente não-D-linked como 'que diabos', conforme atestam os exemplos já fornecidos anteriormente e retomados a seguir:

(30) $* \mathrm{How}_{\mathrm{i}} / \mathrm{Why}_{\mathrm{i}}$ do you regret [that Mary fixed the broken vase $\mathrm{t}_{\mathrm{i}}$ ?

(31) *What the hell $i_{\mathrm{i}}$ do you regret [that Mary bought $\mathrm{t}_{\mathrm{i}}$ on her trip to China]?

(32) ${ }^{*} \mathrm{Como}_{\mathrm{i}} /$ Por que $\mathrm{i}_{\mathrm{i}}$ você lamenta [que a Maria tenha quebrado o vaso chinês $\left.\mathrm{t}_{\mathrm{i}}\right]$ ?

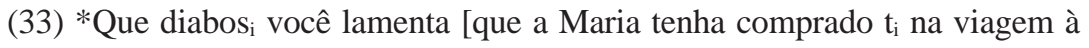
China]? 


\subsection{Complementos nominais e o fato}

Assumi que TopPé um nódulo sentencial que abriga o traço [+ específico] associado aos predicados factivos. Em relação a complementos nominais simples, já mencionei que a imposição de que estes sejam específicos se mostra atuante.

Sem querer me alongar na análise da estrutura interna do DP, quero chamar a atenção para uma proposta de Ihsane e Puskás (2001) que se mostra bastante atraente em relação à análise que proponho para o fenômeno da factividade. As autoras propõem uma estrutura para a periferia esquerda dos nominais que é paralela ao proposto por Rizzi (1997) para a periferia esquerda da sentença. A intenção é distinguir duas projeções funcionais: uma para especificidade e outra para definitude. A projeção que hospeda o traço [+/- definitude] corresponde sintaticamente ao nódulo FinP no sistema de Rizzi (1997), isto é, a projeção mais baixa da periferia esquerda, enquanto o traço [+ específico] caracteriza uma projeção paralela ao nódulo TopP. Dessa maneira, as autoras explicam a possibilidade de sintagmas nominais definidos apresentarem também a leitura não-específica ${ }^{10}$. Essa possibilidade se localiza no conjunto de traços que o artigo definido pode exibir, dadas as checagens feitas. $\mathrm{O}$ artigo definido é gerado em $\mathrm{Def}^{0}$. O traço [+definido] é checado localmente, enquanto o traço [+específico] deve ser checado em TopP, via movimento. Se o artigo definido for a realização do traço [+definido] sem o traço de especificidade, ele permanece em Def ${ }^{0}$. As estruturas são dadas a seguir ((12a,b) no original):

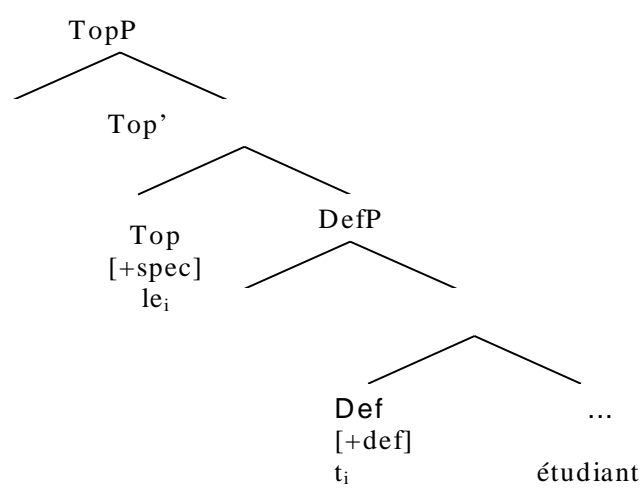

10 Exemplos relevantes apontados no original são:

(i) Jái pris le train.

I have taken the train

'I took the train.' (ii) Jean a raté le bus.

John has missed the bus.

'John missed the bus.' 


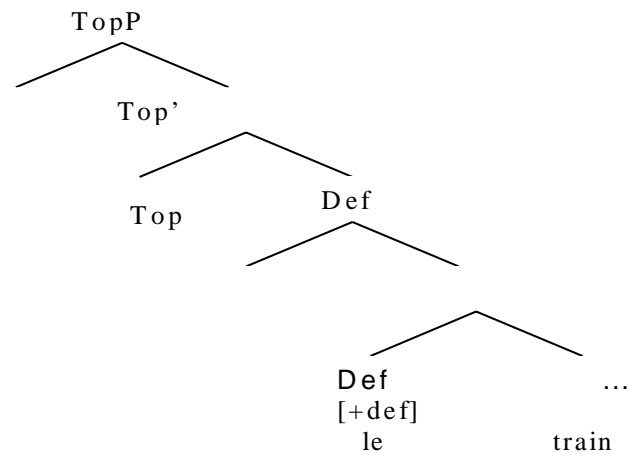

Embora as autoras não definam claramente qual seria a posição de base do artigo indefinido, uma série de projeções funcionais são admitidas abaixo de DefP, as quais abrigariam demonstrativos, numerais, adjetivos. Pode-se supor que o artigo indefinido seria gerado em uma dessas projeções e, uma vez apresentando leitura específica, subiria para o nódulo Top. Chega-se, assim, a um paralelo entre as sentenças complementos de verbos factivos e os complementos simples.

As autoras mencionam, ainda, que um DP [+spec], que apresenta uma leitura de tópico, sofre movimento em LF. Assim, obtém-se as seguintes representações $((31 \mathrm{a}, \mathrm{b})$ no original):

(35) [IP J'ai pris [DP[non-specific] le train]

(36) $[$ Top $[\mathrm{DP}[+$ specific] le train] [IP J'ai pris e]

Essa proposta faz lembrar o mecanismo adotado por Diesing (1992) para explicar a impossibilidade de extração a partir de DPs. ${ }^{11}$

Seja qual for a análise oferecida que explique a impossibilidade de extração a partir de DPs definidos, ela deve ser estendida para alguns dos contextos na Ilha Factiva. A impossibilidade de extração é atestada tanto a

${ }^{11}$ Diesing (1992) propõe, a partir de Heim (1982), o mecanismo denominado the Mapping Hypothesis, pelo qual as representações lógicas de uma sentença podem ser derivadas a partir de representações sintáticas. A estrutura sintática é dividida em duas partes: a cláusula restritiva e o escopo nuclear. Nessa proposta, uma vez que o VP constitui o escopo nuclear da proposição, NPs definidos, sendo pressupostos, devem ocupar uma posição fora do VP. Adicionalmente, ela assume uma restrição que defende que extrações não podem ocorrer a partir de um NP que deverá ser alçado para uma posição fora do VP. Na verdade, após o movimento do NP, haverá uma barreira extra entre o elemento-Qu e o vestígio, constituindo-se, assim, uma violação de Subjacência no âmbito da análise em Barriers (Chomsky 1986).

Apresentar uma análise alternativa que seja mais adequada aos pressupostos minimalistas poderia passar por Hornstein (1995) que, embora assuma com Diesing (1992) que elementos definidos devem estar fora do VP em LF, defende que essa configuração é obtida via movimento para as posições de atribuição de Caso (para os objetos, AgrO, por exemplo) e não pela operação de Quantifier Raising $(Q R)$. Ao se apagarem os elos da cadeia em LF, essa imposição de que definidos estejam fora de VP deve ser atendida. Embora esse 
partir de complementos definidos quanto dos indefinidos específicos seguidos de relativa (estrutura que impossibilita extração independentemente da construção factiva):

(37) Lamentei a venda das estatais pelo governo.

(38) *O que/De quê você lamentou a venda t pelo governo?

(39) Lamentei uma briga que eu tive com meu chefe.

(40) *Com quem você lamentou uma briga que você teve t?

Além de abordar a questão dos sintagmas nominais simples complementos de verbos factivos, é importante também nos determos na construção com o elemento o fato que pode ser seguido por uma sentença encaixada:

(41) Pedro lamentou o fato (de) que Maria tinha brigado com várias amigas. (42) John regretted the fact that Mary had quarreled to several friends.

Quero salientar que a relação entre o objeto direto da sentença matriz e a sentença encaixada não se configura como o de uma complementação. Ormazabal (1995), seguindo Stowell (1981) salienta que a relação temática entre nomes e seus complementos nominais não é a mesma que entre um nome e um argumento sentencial, exemplificando com os sintagmas abaixo ((109a, b) no original):

(43) Their explanation [of the problem]

(44) Their belief/claim [that the problem could be solved]

\section{Ormazabal (1995:138) remete a Stowell (1981):}

unlike the nominal in (43) [minha numeração em toda a citação; MA], the

autor não trate da questão da extração, pode-se especular que, nesse caso, as assunções anteriores nos levariam a uma construção em LF, em que se tem um elemento em posição de especificador, com uma parte do qual a cadeia-QU deve ser formada. Sabemos que extrações a partir de especificadores não são permitidas, mas essa restrição se explica derivacionalmente nas análises de teor minimalista (ver Nunes e Uriagereka (2000), por exemplo), por imposições de linearidade que dizem respeito a PF e lançam mão da noção de Spell-Out. Portanto, algumas especulações adicionais seriam necessárias para se viabilizar uma análise nesses moldes, o que não é o objetivo nesse momento.

Outra alternativa que análises minimalistas têm cotejado diz respeito à possibilidade de considerar também DP uma fase, como o faz Adger (2002), que afirma, no entanto, que somente DPs definidos constituiriam ilhas para a extração, mas não os indefinidos, e que tal se daria pela possibilidade de somente Ds indefinidos permitirem um especificador com traço-QU, através do qual o movimento sucessivo cíclico se efetivaria. Evidência adicional para essa proposta consiste na diferença de comportamento quanto a movimento-QU interno ao DP que se estabelece entre os definidos e os indefinidos (exemplos (87) e (88) no original):

(i) A fierce battle $\sim$ How fierce a battle

(ii) The fierce battle $\sim$ *How fierce the battle 
noun phrase in (44) does not refer to the act of believing or claiming, but rather to the content of the belief or the claim itself. Thus the relation between the noun belief or claim and the tensed clause is not the typical relation observed between a head and its complement, but rather they stand in an apposition relation. The fact that they may show up in an identity relation, as in (45) and (46), suggests that they refer to the same semantic object.

(45) *their explanation was [(of) the problem]

(46) Their belief/claim was [that the problem could be solved]

Analogamente, pode-se dizer que o elemento $o$ fato e a sentença encaixada também estão em uma relação de identidade:

(47) O fato é que a Maria está grávida.

Ambos elementos - o sintagma nominal e a sentença complemento remetem ao mesmo referente. Trata-se de uma sentença equativa. Conforme me chamou a atenção Mary Kato (comunicação pessoal), a característica das sentenças equativas é que sujeito e predicado são definidos.

Assim, nas sentenças factivas com $o$ fato, a especificidade é checada pelo artigo definido desse sintagma, obtendo-se a leitura de conhecido/ pressuposto. Se há uma relação de identidade entre $o$ fato e a sentença complemento, a leitura de pressuposto se estende também à sentença encaixada, que, vou assumir, se traduz em uma estrutura apositiva, nesse caso. É desnecessário salientar que extrações a partir de um adjunto são desautorizadas. Os dados se conformam ao previsto: ${ }^{12}$

(48)*What do you regret the fact [that Mary bought $\mathrm{t}$ on her trip to China]? (49)*O que o Pedro lamenta o fato de [que a Maria tenha feito $t$ ]?

12 Um ponto deve ser enfocado. Extrações de argumentos, no inglês, a partir de sentenças com a presença do pronome it são marginalmente aceitáveis:

(i) *?What do you regret it [that Mary bought on her trip to China]?

A presença do pronome sugere o mesmo tipo de ${ }^{\mathrm{i}}$ estrutura proposto para a sentença com a presença de the fact. Sendo assim, esperar-se-ia a impossibilidade de extração. Jairo Nunes (comunicação pessoal) sugere que a distinção na presença de $i t$ ou de the fact possa ser atribuída ao fato de que o CP seria complemento do primeiro, enquanto está em adjunção ao segundo. Assim, no primeiro contexto, a extração violaria somente definitude; já no caso da presença de the fact, a extração viola definitude e adjunção. Não tenho espaço para explorar aqui as consequiências que tal análise acarretaria.

Deve-se, ainda, salientar a impossibilidade de extração na presença de artigos definidos, conforme atestam os exemplos a seguir (cf. nota 4):

(ii) $* \mathrm{O}$ que o Pedro lamenta o terem-se lançado?

(iii) *O que nós lamentamos o eles terem recebido?

(iv) *Qué lamentas el que María haya comprado en su viaje a China? 


\section{Mapeamento do complemento [+ específico]: a leitura de pressuposto}

Uma das propostas de formalização que pretende capturar as principais propriedades das construções de topicalização se traduz pela adoção da denominada Estrutura da Asserção de Zubizarreta (1998). Trata-se de uma representação abstrata derivada pós-LF na qual as distinções focopressuposição e tópico-comentário são mapeadas. A Estrutura de Asserção é representada contextualmente, ou seja, a estrutura de foco-pressuposição prevê duas asserções ordenadas. Segundo Zubizarreta (1998:4), a primeira asserção $\left(\mathrm{A}_{1}\right)$ traz a pressuposição existencial que pode ser explicitada por uma questão contextualizadora, tendo a forma de uma quantificação existencial, enquanto a segunda asserção $\left(\mathrm{A}_{2}\right)$ representa a principal asserção, na qual se atribui um valor para a variável introduzida pela questão contextualizadora.

(50) $\mathrm{A}_{1}$ : There is an $x$ such that John ate $x$

(51) $\mathrm{A}_{2}$ : The $x$ such that John ate $x=$ the pie

Também a partição tópico/comentário pode ser representada na Estrutura de Asserção da sentença. O tópico da asserção é o sujeito de um predicado proposicional aberto, sendo o predicado proposicional o comentário. A relação tópico/comentário é codificada, portanto, na primeira asserção $\left(\mathrm{A}_{1}\right)$, sendo o tópico o sujeito do predicado proposicional. Para efeitos de ilustração, Zubizarreta fornece o seguinte exemplo ((21) e (22) no original):

(52) $[\mathrm{F}$ Fred $]$ ate the beans.

QC: [What about the beans? Who ate them?]

(53) $A_{1}$ : the beans $y_{y}$ there is an $x$, such that $x$ ate $y$

$\mathrm{A}_{2}$ : the beans $\mathrm{y}$ the $\mathrm{x}$ ( such that $\mathrm{x}$ ate $\left.\mathrm{y}\right)=$ Fred

Assumindo-se que a Estrutura de Asserção da sentença, ao explicitar em termos de escopo a noção tópico/comentário, pode ser responsável por fazer o mapeamento entre a estrutura sintática e o componente de interpretação semântica, explica-se como a leitura de re, de pressuposto, de existência, de fato, é alcançada nas estruturas de verbos factivos que estou assumindo. Assim, DPs complementos específicos como em (54) abaixo poderão ser mapeados como tópicos (ver (55)). Já os não-específicos impedem a leitura de pressuposto ao não se qualificarem como elementos passíveis de se submeterem ao mapeamento para a posição de tópico (56) e (57). Dado que a exigência de especificidade para os complementos de predicados factivos 
não se cumpre, essas sentenças são excluídas. ${ }^{13}$ A possibilidade de mapeamento como tópico também se estabelece quando da presença do elemento o fato, dada sua especificidade, como ilustrado em (58) e (59). No caso de sentenças complemento sem esse elemento, é justamente a estrutura com a presença do nódulo TopP que permite esse mapeamento, conforme ilustram (60) e (61):

(54) Pedro lamentou [DpO resultado das eleições no Rio].

(55) O resultado das eleições no Rio ${ }_{y}$ lexiste um x, tal que x lamentou y

(56) *Pedro lamentou um acidente.

(57) *Pedro lamentou [ст que a paz mundial dependa dos EUA].

(58) Pedro lamentou [DPO fato de que a paz mundial dependa dos EUA].

(59) $\mathrm{O}$ fato de que a paz mundial dependa dos $E \mathrm{~A}_{\mathrm{y}} \backslash$ existe um $\mathrm{x}$, tal que $\mathrm{x}$ lamentou y.

(60) Pedro lamentou [Topque a paz mundial dependa dos EUA].

(61) Que a paz mundial dependa dos $E \mathrm{~A}_{\mathrm{y}}$ lexiste um $\mathrm{x}$, tal que $\mathrm{x}$ lamentou $\mathrm{y}$.

Uma objeção que poderia ser levantada em relação ao mapeamento do complemento como tópico se alicerça sobre a possibilidade de que a QC questione o próprio complemento, o que implicaria que esse se constituísse como foco. ${ }^{14}$ Mas ao explorarmos detalhadamente essa possibilidade, nos deparamos com algumas especificidades, frente às quais, assumo que uma QC pode ter como resposta um foco ou um tópico contrastivo.

Molnár (2001) defende que: "Contrastiveness is not only marked in the field of focusing, but also in combination with topicality". Para diferenciálos, Molnár remete aos traços [+/- exclusivo] e [+/- exaustivo]:

For the focus operator the features [+exclusive] and [+exhaustive] are characteristic expressing that all alternatives are excluded, whereas the contrastive topic contains the combination [+ exclusive] and [- exhaustive], indicating the exclusion of only one or some of the relevant alternatives. The difference between the two exclusion types can thus be described by the opposition of 'strong exclusion' ('all-exclusion') and 'weak exclusion' ('someexclusion').

The information structural value of the weak exclusion is differently judged in literature: it is subsumed either only under focus, called 'Contrafocus' by Kenesei (1989), or only under topic - called 'S-Topic' by Büring (1997), 'ITopik’ by Jacobs (1997).

13 Em relação a (57), é bom lembrar que CP e TopP sentencial se diferenciam justamente porque somente esse último satisfaz a restrição selecional dos predicados factivos, isto é, de que o complemento seja [+ específico].

${ }^{14}$ Agradeço a Mary Kato por ter apontado esse aspecto. 
Essa distinção é notada no exemplo abaixo, em que dois tipos distintos de resposta são fornecidos para uma questão:

(62) O que o Alexandre almoçou?

(63) Feijão, arroz e bife.

(64) O frango.

A resposta (63) constitui o foco, já (64) é um exemplo de tópico contrastivo. ${ }^{15}$ Assim, é importante salientar que a resposta a uma QC pode se dar tanto pela determinação do foco da sentença como de um tópico contrastivo, sobre o qual Zubizarreta (1998) não faz menção.

Em relação ao contexto sob investigação, isto é, a complementação factiva, quero chamar a atenção para o fato de que uma pergunta como (65) a seguir, parece inadequada para ser feita out-of-the-blue. Isto é, esse tipo de pergunta com lamentar implica um contexto apropriado em que o tópico 'coisas lamentáveis' esteja saliente. Logo, nesse caso, respostas que se constituam simplesmente como foco seriam inadequadas, apenas respostas que contrastem/explicitem uma possibilidade, excluindo as demais colocadas em evidência pelo tópico 'coisas lamentáveis', se configurariam apropriadas nesse contexto, isto é, um tópico contrastivo.

\section{(65) ?O que o Pedro lamenta?}

Essa hipótese pode ser corroborada ao compararmos perguntas com o elemento "que diabos" (66) associadas aos contextos apresentados anteriormente. Verificamos que "que diabos" não suporta uma resposta do tipo tópico contrastivo. Curiosamente, a combinação desse elemento com lamentar resulta agramatical, conforme (67) ilustra, corroborando nossa hipótese de que o complemento desses verbos é um elemento passível de ser interpretado como tópico (ou tópico contrastivo), e isso exclui a combinação com um elemento como "que diabos", essencialmente não- $D$-linked, que não admitiria tópicos contrastivos.

(66) A: Que diabos o Alexandre comeu no almoço?

B: Arroz, feijão e bife.

$* \mathrm{O}$ frango.

15 Os contextos são distintos. A primeira resposta é adequada para uma pergunta do tipo out-of-the-blue. Ao chegar em casa após o horário de almoço, a pergunta pode ser feita e implica um pedido de informação, que é fornecido pela resposta em (63). Já o segundo tipo de resposta pressupõe uma situação particular. Imaginemos que sobrou um pouco de fígado do jantar e a mãe de Alexandre pediu à cozinheira que fizesse também um pouco de frango para completar o almoço. Quando essa mãe chega em casa e faz a pergunta em (62), recebe como resposta (64). Esse tipo de resposta pode ser avaliado como um tópico contrastivo. A resposta explícita é: $\mathrm{O}$ frango e não o fígado. Evidentemente, outros acompanhamentos estavam presentes, talvez arroz, feijão, salada, etc. Ou seja, é um contexto de exclusão, mas não de exclusividade. 
Evidência adicional de que o complemento factivo não se constitui como foco vem do basco (predominantemente da região oriental denominada Bizkaian). Nessa região, complementos factivos são preferencialmente acompanhados de um complementizador com marcas morfolológicas distintas. Trata-se de -ena, aparentemente o complementizador -en seguido de um artigo definido $-a$. Artiagoitia (2003:481) fornece o seguinte exemplo ((39) no original):

(68) A: Zer dago argi? / Zer entzun duzu?

What is clear / what hear AUX

What is clear? What have you heard?

(69) B: Amaiak asko ikasten duela.

Amaia.ERG much study.IMPF AUX.ela

(70) B: *Amaiak asko ikasten duena.

Amaia.ERG much study.IMPF AUX.ena

That Amaia studies a lot.

O seguinte comentário é acrescido:

yet many speakers of Westem Basque will prefer -ena if the statement introduced by the complementizer is assumed to be true and is not the real focus of the main sentence (...)

Therefore, sentences introduced by -ena are necessarilly true statements in the mind of the speaker, statements that she or he takes for granted and do not provide new information, consequently, they cannot be the focus of the sentence.

Ou seja, essa variedade do basco mostra claramente que complementos factivos não são adequados como foco.

\subsection{Mapeamento em LF ou pós-LF?}

Embora esteja compartilhando da idéia (Ormazabal 1995) de que as relações de escopo são importantes para que a leitura de pressuposição se efetive, transfiro para a Estrutura de Asserção de Zubizarreta (1998), o mapeamento dessa relação. Minha análise prevê que esse mapeamento se realizará dadas as características peculiares da própria projeção - TopP que hospeda esse complemento e que é requerida por propriedades selecionais do predicado matriz. Além de estabelecer as relações sintático-semânticas comumente atribuídas a essa classe de verbos, a estruturação aqui proposta apresenta a vantagem de equacionar as peculiaridades da questão da extração na Ilha Factiva, ao excluir extração de adjuntos pela impossibilidade de usarem 
[Spec, TopP] como pouso intermediário.

A opção pela Estrutura de Asserção vem ao encontro do questionamento que se levanta em relação a tratar a distinção de dicto/de re - atributivo/ referencial como reflexo de uma interação de escopos em LF, ou seja, como aplicação da regra de Quantifier Raising (QR). Hornstein (1987) e Graça (2002) apontam para a relevância de se tratar o fenômeno da pressuposição existencial como um efeito pragmático. Analogamente, argumento pela adequação de se tratar a pressuposição factual como parte do fenômeno mais geral. Assim sendo, a adoção da Estrutura de Asserção traz a grande vantagem de prescindir da noção de movimento em LF para caracterizar a pressuposição. Ao lançar mão da noção de [especificidade] associada ao conceito de tópico, garanto o mapeamento para a leitura de pressuposição, via Estrutura de Asserção, no componente semântico/pragmático, pós-LF.

É ainda importante salientar que, se é lícito incorporar a noção de Estrutura de Asserção no modelo de gramática adotado, conforme defende Zubizarreta (1998), então esperar-se-á que toda sentença se submeta a ela. Assim, podemos prever uma redundância em relação às análises que propõem um movimento em LF para as expressões definidas, uma vez que essas já serão possivelmente mapeadas como tópicos pela Estrutura de Asserção. A análise aqui proposta prescinde dessa redundância.

\section{Evidência adicional}

Uma evidência de que a sentença encaixada se comporta como um tópico (sentencial) é o fato de tópicos comuns (nominais) não serem licenciados nessas configurações:

(71) *John regrets that John, Mary likes.

(72) *Pedro lamenta que o ex-namorado, a Maria encontrou (ele) no cinema.

Rizzi (1997:297) contrapõe FocP a TopP, salientando que o foco é único enquanto TopP pode apresentar múltiplas projeções. Isso se dá uma vez que, de um lado, temos uma articulação entre foco e pressuposição e seria incongruente um foco dentro do pressuposto. Por outro lado, em relação a TopP, a articulação se dá entre tópico e comentário e nada impede que, dentro do comentário, um novo tópico seja colocado em relevo.

$\mathrm{O}$ nódulo TopP aqui proposto, no entanto, é um pouco diferente, já que se trata de um TopP sentencial, isto é, em que a sentença encaixada como um todo constitui o tópico. Sendo assim, assumir um tópico dentro dessa sentença não se configuraria como um tópico dentro do comentário, mas sim um tópico dentro do tópico. Assim como discutido em relação à recursividade de FocP, também neste caso, teríamos uma incongruência interpretativa, o que é 
evidenciado pela impossibilidade de (71) e (72). ${ }^{16}$

\section{O caráter excepcional do grego moderno}

O grego moderno apresenta dois complementizadores: oti e pu. O primeiro é comum para introduzir sentenças encaixadas (geralmente extrações são admitidas a partir de sentenças introduzidas por oti). $P u$ é encontrado em relativas, clivadas, exclamativas, além de introduzir complementos de verbos psicológicos e de percepção, e os factivos.

A comparação entre português, por exemplo, e grego moderno poderia sugerir que a sentença (73) deveria ser equiparada à sentença (74), sem a presença de $o$ fato. No entanto, o que quero apontar é que o comportamento de (73), em grego moderno, é análogo ao apresentado por sentenças como (75), em português.

(73) *Pjon lípase pou sinandise o Yanis?

Who regret-2.SG that met-3.SG John-NOM

Who do you regret that Y. met?

(74) $\mathrm{O}$ que você lamenta [que a Maria tenha comprado t $t_{\mathrm{i}}$ na viagem à China?

(75) *O que i $_{i}$ você lamenta o fato de [que a Maria tenha comprado $t_{i}$ na viagem à China?

O que as sentenças têm em comum é a presença de um traço [+ definido], ou seja, seguindo Roussou (1994), assumo que pu, em grego moderno, é um complementizador com o traço [+definido]. A definitude apresentada pela sentença (73) explica a impossibilidade de extração.

Roussou (1994) defende que pu apresenta o traço [+ definido] uma vez que não pode ser precedido por um artigo definido, contrariamente a oti que aceita o artigo ((40a,b) e (44) e (45) no original):

(76) To oti perase tis eksetasis me efxaristise.

The-nom that passed-3s the exams me pleased-3s

'the fact that she passed the exams pleased me.'

(77) *To pu perase tis eksetasis me efxaristise.

The-nom that passed-3s the exams me pleased-3s

'the fact that she passed the exams pleased me.'

16 Alguns falantes julgam (72) aceitável, o que se deve, possivelmente, à possibilidade de uma leitura de tópico contrastivo se efetivar nesses casos. Conforme mencionado, tópicos dentro de tópicos sentenciais seriam inaceitáveis dada uma incongruência interpretativa, a qual poderia ser burlada pela presença, no entanto, de um tópico contrastivo. De qualquer maneira, vale apontar a nítida distinção entre (72) e a sentença a seguir:

(i) Pedro disse que o ex-namorado, a Maria encontrou (ele) no cinema. 
(78) $*[$ (to) pu efighe $]$ me stenaxorese.

The that left-3s me-upset-3s

(79) Me stenaxorese [pu efighe]

Me-upset-3s that leeft-3s

'It upset me that he left.'

(80) [To oti efighe] me stenaxorese.

The that left-3s me-upset-3s

'That he left upset me.'

Assim, Roussou compara a impossibilidade de artigo $+p u$ em paralelo à impossibilidade de uma sequiência de Ds para um NP, como em (81) a seguir:

(81) *The this book

Em suma, estruturas com pu são estruturas definidas. Se levarmos em consideração que DPs definidos criam um ambiente a partir do qual a extração é impossível, explica-se o comportamento de ilha forte atestado nessa língua, em paralelo a sentenças como (82) (Chomsky 1973). Seja qual for a análise proposta para a impossibilidade de extração a partir de complementos definidos (veja a discussão na nota 11), trata-se do mesmo fenômeno.

\section{*Who did you see the picture of?}

Em suma, o comportamento peculiar do grego moderno é atribuído à presença de um complementizador particular com marcas morfológicas de definitude. Evidência adicional de que o complementizador especial implica a leitura de pressuposto e a impossibilidade de extração é o fato de alguns verbos admitirem o complementizador oti, sendo que nesses casos, conforme esclarece Varlokosta (1994:246, nota 8), o padrão de extração exibe a distinção argumento/adjunto:

The pattern of extraction is more complicated (...). With ambiguous predicates such as thimame 'remember', pou-clauses block any extraction but oti-clauses result in an argument vs. adjunct asymmetry with respect to extraction, essentially patterning like Spanish or Italian factive complements.

\section{Conclusão}

Este artigo tratou das construções factivas buscando explicitar a conexão entre o aspecto semântico do fenômeno, isto é, a interpretação pressuposicional deflagrada por esses predicados, e as possibilidades de extração em sentenças complexas, que caracterizam, tradicionalmente, a Ilha Factiva como uma ilha fraca, ou seja, em que argumentos são passíveis de extração, mas não 
adjuntos.

A preocupação central esteve no mapeamento entre a sintaxe e a semântica e a maneira como aspectos semânticos podem fazer parte das propriedades codificadas pelo sistema computacional. Aproximou-se a noção de pressuposição estrutural deflagrada pelos predicados factivos à noção de especificidade, propondo-se que esses predicados selecionam um complemento do tipo [+ específico]. Assumiu-se a proposta de expansão do CP de Rizzi (1997) que admite nódulos do tipo Tópico e Foco, propondo-se a instanciação de um nódulo TopP sentencial acima de $\mathrm{CP}$, condizente com a noção de especificidade e a partir da qual se estabelece a distinção entre leitura de dicto e de re para sentenças complemento.

A adoção da idéia de nódulos que podem ser interpretados como tópicos permitiu defender que o mapeamento da construção factiva se submete à Estrutura de Asserção de Zubizarreta (1998), efetivando-se, por fim, a leitura de pressuposição.

A possibilidade de satisfação do traço [+ específico] por diferentes estruturas resulta interessante na medida em que uma observação detalhada dos padrões de extração mostra um quadro bem mais complexo do que a simples distinção argumentos/adjuntos, comumente atribuída às ilhas fracas, preveria.

Em suma, a análise aponta para a não-biunivocidade entre seleção semântica (s-seleção) e seleção categorial (c-seleção). Isso se evidencia, por exemplo, pela presença de complementos definidos nominais em oposição aos sentenciais. Mesmo aqui há uma distinção em relação à presença do elemento $o$ fato. Quando este elemento está presente na Numeração de uma dada derivação, ele é capaz de checar o traço [+ específico]. A sentença que se segue é tomada como um aposto, à qual se estende o caráter de pressuposição. Conforme foi verificado, qualquer tipo de extração é desautorizada a partir dessa sentença. No caso de complementos simples, ao se assumir Ihsane e Puskás (2001), a presença do nódulo TopP, responsável por garantir a especificidade do sintagma nominal, permite um mapeamento automático na Estrutura de Asserção, desse elemento, como pressuposto. A impossibilidade de extração decorre da presença da projeção DP (Chomsky 1973, Diesing 1992). As sentenças complemento sem a presença de o fato foram analisadas como projeções de TopP. A presença de TopP sentencial garante a satisfação da seleção de complementos [+ específicos], sendo que o traço [+ específico], em Top ${ }^{0}$, é checado, via o movimento do complementizador que, da sentença encaixada, para o núcleo de TopP, estendendo-se, assim, a especificidade para toda a sentença. Nesse tipo de construção, constata-se a distinção argumento versus adjunto em relação às possibilidades de extração. [Spec,TopP] funciona como válvula de escape 
apenas para o movimento de elementos- $Q u$ compatíveis com a posição de tópico, ou seja, somente argumentos, mas não adjuntos são extraídos. ${ }^{17}$ Essa restrição imposta pela presença do nódulo TopP é também responsável pelas restrições de leitura que os elementos passíveis de extração sofrem, conforme discutido na seção 2 .

Em suma, análises mais recentes (Nunes \& Uriagereka 2000, Stepanov 2001) a partir do Programa Minimalista (Chomsky 1995, 2000) vêm desafiando uma tendência, que havia se firmado em relação às restrições de ilha no arcabouço de Princípios e Parâmetros, mais especificamente desde o trabalho pioneiro de Ross (1967) e contribuições posteriores de Chomsky (1973, 1986), em que basicamente se assumia que as restrições de ilha representavam fundamentalmente um único fenômeno, solidificando-se uma estratégia de tratamento em que se examinava, em termos de limites/barreiras, a possibilidade de estabelecimento da relação de dependência entre o elemento deslocado e sua respectiva lacuna. A análise da Ilha Factiva aqui apresentada se alinha às análises que desafiam essa unificação de tratamento.

E-mail: marina_augusto@uol.com.br Recebido em abril de 2003 Aprovado em julho de 2003

\section{REFerÊnCias Bibliográficas}

AdGER, D. 2002. Core syntax: a minimalist approach. Ms. University of York.

Aoun, J.; N. Hornstein; D. Lightfoot \& A. Weinberg. 1987. Two types of locality. Linguistic Inquiry 18:537-77.

Artiagoitia, X. 2003. Complementation (noun phrases). In: J. I. HualdE \&

J. Ortiz de Urbina (eds.) A grammar of Basque. Berlin: Walter de Gruyter. Augusto, M. R. A. 1994. Fatores envolvidos na extração dos adjuntos- $Q$.

Dissertação de Mestrado, Universidade Estadual de Campinas. 2003. Padrões de extração em estruturas factivas. Tese de Doutorado, Universidade Estadual de Campinas.

Beghelli, F. \& T. Stowell. 1997. Distributivity and negation: the syntax of each and every. In: A. Szabolcsi (ed.) Ways of scope taking. The Netherlands: Kluwer Academic publishers.

Сномsкy, N. 1973. Conditions on Transformations. In: S. R ANDERson e P. KIPARSKY (eds.) A Festschrift for Morris Halle. New York: Holt, Reinhart

\footnotetext{
${ }^{17}$ Sentenças reduzidas e complementos sentenciais no modo subjuntivo são tratados, adotando-se o mesmo arcabouço teórico aqui apresentado, em Augusto (2003).
} 
and Winston, Inc.

.1986. Barriers, MIT Press, Cambridge, Massachusetts.

1995. The minimalist program. Cambridge, Massachusetts: MIT Press.

2000. Minimalist Inquiries: the framework. In: R Martin, D. Michaels \& J. UriagereKa (eds.) Step by step. Essays on minimalist syntax in honor of Howard Lasnik. Cambridge, Mass.: 89-155.

Cinque, G. 1990. Types of A'Dependencies., Cambridge, Massachusetts: The MIT Press.

Cyrino, S. M. L. 1994. O objeto nulo no português do Brasil - um estudo sintático-diacrônico. Tese de doutorado, IEL/UNICAMP.

Diesing, M. 1992. Indefinites. Cambridge, Mass: MIT Press.

ENÇ, M. 1991. The semantics of specificity. Linguistic Inquiry 22.1:1-25.

Fodor, J. D. \& I. SAG. 1982. Referential and quantificational indefinites.

Linguistics and Philosophy 5: 355-398.

GRAÇA, A. S. 2002. Referência e Denotação: duas funções semânticas

irredutíveis. Disputatio 12: 3-21. [Online] Disponível: http://

www.disputatio.com/articles/012-1.pdf

Hegarty, M. 1992. Familiar complements and their complementizers: on some determinants of A'-locality. Ms. University of Pennsylvania.

HeIm, 1982. The semantics of definite and indefinite noun phrases. Tese de doutorado. University of Massachusetts.

HeYcock, C. 1995. Asymmetries in Reconstruction. Linguistic Inquiry 26: 547-570.

HoRnsteIn, N. 1984. Logic as Grammar: an approach to meaning in natural language. Cambridge: MA MIT Press.

1995. Logical Form: from GB to Minimalism. Cambridge: Blackwell.

HuAng, C.-T.J. 1982. Logical relations in Chinese and the theory of grammar. Doctoral Dissertation. Cambridge, Mass.: MIT.

Insane, T. e G. Puskás. 2001. Specific is not definite. Generative Grammar in Geneva 2: 39-54.

Kiparsky, P. \& C. Kiparsky. 1971. Fact. In: D. Steinberg \& L. Jakobovits (eds.) Semantics. Cambridge: Cambridge University Press. [Reprinted from M. Bierwisch \& K. Heidolph (eds. 1970) Progress in Linguistics. Mouton, The Hague].

Manzini, R. 1997. A minimalist theory of weak islands. Ms. [Publicado em: Syntax and Semantics 29. The Limit of Syntax. Academic Press:185-209].

Melvold, J. 1991. Factivity and Definiteness. In: L. Cheng \& H. Termidash (eds.) MIT Working Papers in Linguistics, 15: 97-117.

Molnár, V. 2001. Contrast from a contrastive perspective. In: I. KorBAYON 
\& M. SteEdman (eds.) Proceedings of ESSLLI Workshop on Information

Structure. Discourse Structure and Discourse Semantics: 99-114.

Nunes, J. \& J. UriagereKa. 2000. Cyclicity and extraction domains. Syntax 3: $20-43$.

Ormazabal, J. 1995. The syntax of complementation: on the connection between syntactic structure and selection. Tese de doutorado. University of Connecticut.

Pesetsky, D. 1987. Wh in situ: movement and unselective binding”. In: E.

Reuland, A. ter Meulen (eds.) The representation of (in)definiteness, MIT Press, Cambridge, Mass.

RAPOSO, E. 1987. Case Theory and Inf-to-Comp: the inflected infinitive in

European Portuguese. Linguistic Inquiry 18:1: 85-110.

Rızzi, L. 1990. Relativized Minimality. Cambridge, Massachusetts: The MIT Press.

. 1997. The fine structure of left periphery. In: L. HAEgEman (ed.) Elements of grammar. Kluwer Academic Publishers: 281-337.

. 2000. Reconstruction, weak islands sensitivity, and agreement. Ms.

Università di Siena.

Ross, J. R. 1967. Constraints on Variables in Syntax. Tese de doutorado, MIT.

Roussou, A. 1994. The syntax of complementisers. Tese de doutorado.

University College London.

Stepanov, A. 2001. The end of CED? In: K. Megerdeomian \& L.A. Bar-el

(eds.) WCCFL 20 Proceedings, Sommerville, Ma: Cascadilla Press.

Stowell, T. 1981. The origin of phrase structure. Tese de doutorado. MIT. SzABOLCSI, A. \& F. ZwARTs. 1992-3. Weak islands and an algebraic semantics

for scope taking. Natural Language Semantics 1: 235-84.

VARLOKOSTA, S. 1994. Factive complements in Modern Greek. University of Maryland Working Papers in Linguistics 2: 238-258.

Zubizarreta, M. L. 1982a. On the relationship of the lexicon to syntax. Tese de doutorado, MIT.

. 1982b. Theoretical implications of subject extraction in Portuguese.

The Lingusitic Review 2,1: 79-96.

. 1998. Prosody, focus and word order. Cambridge, MA: The MIT Press. 